\title{
ORT_09 - Vaccination status of undergraduate health science students: a matter of great concern
}

Claudia Lamarca Vitral ${ }^{1}$; Caio Henrique da Silva Teixeira ${ }^{1 *}$; Victor Mendel da Silva Mello ${ }^{1}$; Paula Salgueiro Xavier $^{1}$; Gina Peres Lima dos Santos ${ }^{1}$; Sandra Cardoso Fonseca ${ }^{1}$; Silvia Maria Baeta Cavalcanti ${ }^{1}$. ${ }^{1}$ UFF - Universidade Federal Fluminense.

Introduction: Health care workers (HCWs) are more at risk of exposure to and of possible transmission of vaccine-preventable diseases. Hence, the early assessment of their vaccination status, ideally during undergraduate years, is of utmost importance.

Objective: Evaluation of vaccination coverage and knowledge about vaccines, associated infectious diseases and behavior regarding protection among health science undergraduate students.

Methodology: A total of 478 students from the two first years of Medicine, Biomedicine, Nursery, Pharmacy, Dentistry and Biology courses of Universidade Federal Fluminense (UFF) answered a selfadministered form with questions regarding vaccines and vaccine-preventable diseases. Vaccination records were also checked for: tetanus, diphtheria and pertussis (TDP), tetanus and diphtheria (Td), hepatitis B, measles, mumps and rubella (MMR), influenza, varicella and meningococcal vaccines.

Results: Only $31.2 \%$ of students informed that their vaccination record had been previously requested, although $68.6 \%$ reported having been advised at some point about the need for vaccination. Few students reported having had vaccine-preventable diseases, except for mumps $(7.1 \%)$ and varicella $(65 \%)$. Only $24.3 \%$ of participants knew which vaccines should be updated for HCWs, with a higher rate of correct answers for Medicine, Biomedicine and Biology students $(p=0.000)$. Immunization schedules for vaccines covered by SUS (Brazilian Health System) and indicated for HCWs (hepatitis B, MMR, Td, influenza), were unknown by most students, except for the flu vaccine. When analyzing vaccination awareness by course, the highest margin of correct answers was observed among Nursery and Dentistry students $(\mathrm{p}=$ 0.000 ), coinciding with the report of a higher exposure to potentially contaminated material (Nursery $60.4 \%$, Dentistry $44.4 \%, p=0.016$ ). It is a matter of great concern that only $5.2 \%$ of all the students had completed the vaccination schedule recommended for HCWs, although $39.1 \%$ had the misperception of being updated. When considering only vaccines offered free of charge by SUS, the rate of students with updated vaccination schedule raised to $26.2 \%$, with a difference favorable to Pharmacy $(35 \%)$ and Nursery $(33 \%)$ courses $(\mathrm{p}=0.001)$. Vaccines with the lowest coverage were influenza $(43.1 \%)$ and $\mathrm{Td}$ $(62.1 \%)$. Among students who were aware of the Td booster every 10 years, $70 \%$ were indeed protected $(p=0.037)$. The same was observed in relation to the flu vaccine, with a higher rate of vaccine coverage among students who are aware of the annual revaccination scheme.

Conclusion: The study showed an overall low vaccination coverage among health science students along with a poor perception about vaccination schedules. These results are worrisome, considering that these future HCWs should be prepared as professionals for guiding the population in the use of vaccines to prevent infectious diseases. To overcome this deficiency, the study of vaccine-preventable diseases and vaccination should be envisaged and deepened as part of the health science courses curricula.

Keywords: vaccination status; university students; health science undergraduate courses 